\title{
EL CÍRCULO DEL TIEMPO
}

-Observaciones acerca de las relaciones entre sujeto y tiempo en las "Lecciones de la fenomenología de la conciencia interna del tiempo"

de E. Husserl*-

\section{Prof. Dr. Ángel E. Garrido-Maturano**}

\section{Introducción}

Se suele decir que pasa el tiempo, pero ¿es el tiempo el que pasa o los que pasan son los objetos en el tiempo? $¿ \mathrm{O}$ acaso en realidad lo que verdaderamente pasa no es ni el uno ni los otros, sino nosotros: nuestra conciencia de los objetos? ¿Es el tiempo un rasgo inherente al mundo o nosotros somos el tiempo? Todas estas preguntas apuntan a una y la misma cuestión: a la de la naturaleza de la relación entre tiempo cósmico y conciencia del tiempo, o, dicho más llanamente, entre tiempo del alma y tiempo del mundo. Edmund Husserl es, entre los filósofos que se han ocupado del tiempo, quizás aquel que con mayor tenacidad y ahínco ha luchado por hacer visibles aquellos estratos más profundos de la conciencia en los que, según su perspectiva, se constituye la relación entre la subjetividad y el tiempo. Su convicción, contra Kant que había declarado la imposibilidad de percibir el tiempo en sí mismo, fue la de que, ahondando en las profundidades de la conciencia, era posible hacer visible el tiempo mismo. Fiel a su método fenomenológico, que pone entre paréntesis "todos los presupuestos trascendentes acerca de lo que existe"1 para atenerse a lo que aparece como tal a la conciencia (fenómenos), esto es, a las vivencias,

\footnotetext{
* Las Lecciones se citan de acuerdo con la siguiente edición española: Edmund Husserl, Lecciones de Fenomenología de la conciencia interna del tiempo, trad. A. Serrano de Haro, Trotta, Madrid, 2002; sigla: LFT Además en cada caso se consigna entre paréntesis la ubicación del pasaje citado en la edición de las obras completas de Husserl: Edmund Husserl , Gesammelte Werke-Husserliana, Vol. X, Zur Pbänomenologie des inneren Zeitbewußtseins (1893-1917), hrsg. von R. Boehm, Dordrecht-BostonLondon, Kluwer Academic Publisher, 1966; sigla Hua X.

** Investigador CONICET, Profesor de la Universidad Católica de Santa Fe y de la Universidad Nacional del Nordeste (Resistencia). hieloypuna@hotmail.com ${ }^{1} L F T$, p. 26 (Hua X, p. 4).
} 
"dudar de las cuales sería absurdo"2, Husserl excluye cualquier convicción a propósito del tiempo objetivo. De acuerdo con su punto de vista fenomenológico, dirige sus análisis a la vivencia de la temporalidad, esto es, al tiempo inmanente al curso de la conciencia, en el cual, como veremos, cree hallar el origen primigenio también del tiempo objetivo. Esta orientación subjetiva ubica a la fenomenología de Husserl dentro de la corriente agustiniana, que centra sus investigaciones acerca del tiempo en la vivencia de la propia temporalidad del alma, y lo enfrenta a la tradición aristotélica, que define al tiempo objetivamente como la medida del movimiento. Ni el derrotero de las estrellas en el cielo ni ningún otra marcha pueden ser llamados tiempo, pues tales movimientos sólo pueden servir para medirlo, pero ellos mismos presuponen la experiencia o conciencia del curso del tiempo. Es alli donde se hallaría la raíz misma de cronos. Sin embargo, en primer lugar, habría que preguntarse si es realmente posible reducir el tiempo del mundo, el tiempo objetivo, a la conciencia del tiempo. En segundo lugar, y correlativamente con la pregunta anterior, habría que preguntarse también si fenomenológicamente es posible determinar los rasgos de la conciencia subjetiva del tiempo de un modo tal que ella se constituya en la fuente originaria de un tiempo objetivo: lineal, homogéneo e irreversible. En otros términos, habría que preguntarse si resultan de la automostración del curso mismo de la conciencia las características formales esenciales que luego encontramos en el tiempo objetivo. Y finalmente habría que preguntarse si realmente está dado al hombre, como pretende Husserl en las Lecciones, mostrar el tiempo mismo. Estas preguntas vertebran la problemática de la presente relectura de la obra de Husserl y anuncian sus tres objetivos. En primer lugar mostrar la excedencia del tiempo del mundo en relación con la temporalidad de la conciencia. En segundo lugar, determinar hasta qué punto la descripción husserliana de la forma del flujo de la conciencia absoluta del tiempo no presupone el tiempo objetivo que pretende constituir y, en consecuencia, hasta qué punto no se cierne sobre la investigación husserliana el riesgo de la circularidad. Finalmente en tercer lugar someter a análisis crítico la idea husserliana de que por medio del método fenomenológico el tiempo en sí mismo puede ser aparecer y ser objeto de descripción. Para alcanzar dichos objetivos seguiremos el hilo

${ }^{2}$ LFT, p. 26 (Hua X, p. 5). 
conductor de las propias Lecciones. En una primera etapa reconstruiremos esquemáticamente los puntos relevantes para nuestra problemática del análisis de la conciencia del tiempo y de sus protoactos y actos reproductivos, que Husserl despliega en la segunda sección de la obra. En una segunda desarrollaremos las dificultades que padece la sección tercera, referida a los niveles de constitución del tiempo, para acceder a la automostración del tiempo en el fluir de la conciencia y fundar en dicha automostración las características formales del tiempo objetivo. Finalmente en una tercera etapa haremos mención a la aporética que se cierne no sólo sobre las Lecciones sino sobre toda investigación que pretenda ya sea reducir uno a otra tiempo objetivo y temporalidad de la conciencia, ya sea asirlos independientemente. En tanto la investigación, así delineada, no pretende ser un examen histórico-filológico del conjunto del pensamiento de Husserl sobre la cuestión del tiempo, sino que se ciñe a la problemática filosófica abierta por la perspectiva de las Lecciones, se me permitirá referir exclusivamente a este texto los análisis siguientes sin considerar en detalle la totalidad de la obra de Husserl al respecto.

\section{Análisis fenomenológico de la temporalidad de las vivencias}

\subsection{El tiempo inmanente}

En las Lecciones Husserl comienza recordando un principio fundamental de la fenomenología, a saber: ni la realidad del mundo natural ni la realidad del tiempo como una característica esencial a ese mundo son dato cierto ninguno. La fenomenología no admite como dato evidente e indisputable la existencia de cosas que duran, sino sólo la conciencia de que las cosas duran. Consecuentemente la fenomenología admite de entrada la existencia de un tiempo, pero no se trata del tiempo del mundo, sino del tiempo interno del curso de la conciencia. En otros términos: el único tiempo del que hay evidencia y que, por tanto, ha de ser tomado como punto de partida de todo análisis del tiempo es, para Husserl la duración inmanente a las propias vivencias de la conciencia ${ }^{3}$. Fenomenológicamente dado está pues el decurso de los momentos de la vivencia y es la conciencia

3 "Que la conciencia de un suceso sonoro, de una melodía que estoy ahora mismo oyendo, muestra una sucesión, de ello tenemos una evidencia que hace absurdas toda duda y toda negación" LFT, p. 26 (Hua X, p. 5). 
de este decurso aquello que nos proporciona nuestras sensaciones o "datos sentidos" específicamente temporales. Pero tales datos de sensación no son propiamente intencionales, no constituyen ningún objeto trascendente y se reducen a una mera afección que siento o padezco. Por ello tales datos nada tienen que ver con el tiempo de los objetos del mundo. "Por análisis fenomenológico no cabe hallar el más mínimo rastro de tiempo objetivo"4. Ahora bien, el punto de partida elegido, a saber, reducir el análisis del tiempo al único en principio indudable: el tiempo inmanente al decurso de las vivencias de la conciencia, no significa que la desconexión del tiempo objetivo sea definitiva, ni que la fenomenología se desinterese de él o ignore el tiempo objetivo. Muy por el contrario, en el meollo de la preocupación de Husserl está poder responder a la pregunta por el origen del tiempo sin más, es decir, por el origen del propio tiempo objetivo, sólo que el pensador dirige esta pregunta a las configuraciones primigenias de la conciencia del tiempo, en las cuales cree ver "las fuentes originarias de todas las evidencias relativas al tiempo"5. Si a Husserl le interesa el tiempo inmanente, las vivencias de tiempo, es porque en la configuración de estas vivencias se mientan los datos objetivamente temporales. Es en la constitución de las vivencias temporales inmanentes donde habría que buscar, pues, el origen de las características formales a priori constitutivas de la objetividad temporal. En síntesis: si se investiga el tiempo inmanente no es para quedarse en el plano de la inmanencia, sino para encontrar la fuente en la que se origina el tiempo trascendente; y no sólo su origen, sino su modo especifico de darse y su legalidad formal a priori ${ }^{6}$.

Este punto de partida, considerado por Husserl evidente, resulta, sin embargo problemático en un doble sentido. Primeramente es cuestionable

\footnotetext{
${ }^{4}$ LFT, p. 27 (Hua X, p. 6).

${ }^{5}$ LFT, p. 31 (Hua X, p. 0).

${ }^{6}$ Husserl lo declara expresamente: "Nosotros buscamos hacer claridad sobre el apriori del tiempo explorando la conciencia del tiempo, sacando a la luz su constitución esencial y poniendo de manifiesto los contenidos de aprehensión y los caracteres de acto específicos, en su caso, del tiempo, a los que pertenecen por esencia las leyes aprióricas del tiempo. Pienso naturalmente en leyes de índole tan consabida como las siguientes: que el orden temporal fijo es una serie bidimensional infinita; que dos tiempos diferentes nunca pueden ser simultáneos; que su relación es no simétrica; que en el tiempo rige la transitividad; que todo tiempo tiene un tiempo anterior y otro posterior, etc." LFT, p. 31 (Hua X, p. 9).
} 
que sólo el tiempo subjetivo y no también el objetivo sea evidente. Si la vida de la conciencia, si el discurrir de sus vivencias vuelve absurdo dudar de la existencia de un tiempo inmanente, de un antes y después de las vivencias, del mismo modo- y aunque parezca una obviedad decirlo -la muerte del cuerpo propio, de la cual también sería absurdo dudar, y que no es una vivencia de la conciencia, sino el ya no más (determinación temporal) del discurrir de las vivencias, torna evidente la objetividad del tiempo con independencia de todo contenido inmanente. En efecto, la muerte introduce una realidad que excede todo acto de conciencia e instaura un antes y un después de la conciencia y no en la conciencia. Dicho en otros términos: el antes y el después, las fases de tiempo, que la muerte en cada caso impone testimonian una continuidad en el tiempo que excede el flujo de cada una de las conciencias, pero que no por ello les es extraño, pues la muerte lo es precisamente de cada una de las conciencias. De este modo el tiempo objetivo, si no se quiere dudar de la muerte por el hecho de que no constituye un contenido inmanente ${ }^{7}$, introduce un instante discontinuo respecto del flujo de la conciencia, sobre la base del cual el tiempo se conforma como una continuidad a través de rupturas, precisamente a través de la ruptura de cada conciencia individual. El primer punto problemático, por tanto, de la desconexión del tiempo objetivo y de la reducción de la evidencia del tiempo al tiempo subjetivo está dado por el desconocimiento de la excedencia de este tiempo objetivo por sobre el subjetivo, cuya evidencia la muerte se encarga de manifestar. Pero no sólo es cuestionable la desconexión del tiempo objetivo y, por tanto, su supuesta no evidencia, sino que en modo alguno es evidente $-\mathrm{y}$ el punto de partida para el análisis fenomenológico debe estar dado sólo por datos indisputables- que la legalidad a priori del tiempo objetivo, esto es, su homogeneidad, linealidad, infinitud, irreversibilidad, transitividad, etc., resulte de la constitución esencial de la conciencia del tiempo. Que el curso del tiempo objetivo, que, además, hemos desconectado por exigencias metódicas, y el curso de las vivencias inmanentes sean isomorfos y que, por tanto, la legalidad a priori del discurrir de las vivencias guarde la misma estructura que le adjudicamos al tiempo objetivo antes de la desconexión, no es un dato fenomenológico, sino un supuesto. Ricoeur tiene razón cuando afirma que Husserl "no puede

7 Sí puede ser considerado un contenido inmanente el morir, la relación con la propia muerte, pero no la muerte en sí, que es esencialmente diferente y posterior a los modos de vivir la relación con ella. 
dejar de admitir, al menos al comienzo de su empresa, cierta homonimia entre el 'curso de la conciencia' y el 'curso objetivo del tiempo del mundo' (...) como si el análisis del tiempo inmanente no pudiera constituirse sin repetidos préstamos del tiempo objetivo desconectado"8. Ciertamente Husserl necesita de estos préstamos para elaborar una hilética temporal de la conciencia, es decir, para describir la forma de los datos temporales sentidos o sensaciones temporales. En efecto, para que esta hilética no quede vacía, Husserl debe interpretar la forma de darse de los datos temporales inmanentes de acuerdo con las aprehensiones en las que aparece lo temporal en sentido objetivo. Pero cabría preguntarse, en primer lugar, si, como pretende Husserl, "la percepción de la duración" tiene su origen en "la duración de la percepción"; y, aún con más énfasis cabría preguntarse, si es la legalidad en el orden del tiempo sentido, es decir, la legalidad de la duración inmanente, la que sirve de base para la constitución del tiempo objetivo, esto es, del modo en que duran los objetos, o si, por el contrario, son los caracteres del tiempo objetivo aquellos a partir de los cuales se intenta interpretar el tiempo sentido inmanentemente. Si este fuera el caso, el tiempo sentido en la inmanencia de la conciencia no sería propiamente "tiempo sentido", no se trataría de una pura hilética temporal de la conciencia, sino que habría ya implícita en la descripción de la conciencia última del tiempo una cierta noética constitutiva de los data fenomenológicos temporales regida por la experiencia que se pretende haber desconectado. Cabría finalmente preguntarse también $-y$ más adelante volveremos sobre el tema- si la hilética temporal de la conciencia, para no quedar vacía, debe recurrir a las determinaciones del tiempo objetivo conocidas antes de la desconexión, o si, el modo inmanente de sentir el tiempo puede implicar variantes respecto de tales determinaciones. Ambas preguntas cuestionan la absoluta evidencia del punto de partida husserliano y colocan bajo sospecha la pretensión de encontrar en la temporalidad inmanente el origen y la fuente de la legalidad a priori del tiempo sin más. Teniendo constantemente en cuenta esta aporética relación entre tiempo de la conciencia y tiempo del mundo nos adentraremos en el análisis husserliano de la conciencia del tiempo.

${ }^{8}$ Ricoeur, Paul, Tiempo y narración III. El tiempo narrado, trad. Agustín Neira, Siglo XXI, Madrid/México, 1996, p. 664; Sigla: TN III. 


\subsection{La serie retención, impresión, protención}

Para su análisis de la conciencia del tiempo, Husserl toma como ejemplo la duración de un sonido. No se trata del sonido como realidad del mundo físico (onda sonora) ni como objeto interno del mundo percibido (nota de la melodía), sino como puro dato hilético, como mera sensación inmanente que dura y que constituye el material, por así decir, con lo que, por un acto de aprehensión, la conciencia constituirá el objeto interno que denominamos nota musical. El sonido emerge en un instante determinado para luego hundirse y en el instante siguiente ser reemplazado por un nuevo sonido incipiente. Pero que el primer sonido se hunde no significa que se disuelve en la nada absoluta (si así fuera entre un sonido y otro no habría continuidad ninguna y la melodía propiamente no duraría, no se podría explicar el pasaje de un sonido a otro), sino que en el nuevo presente junto con la percepción del segundo sonido continúa presente el primero a modo de recuerdo, aunque ya no sea percibido en carne y hueso. "En este su hundimiento en el pasado yo lo 'mantengo' aún sujeto, lo tengo en una 'retención', y mientras la retención pervive, el sonido posee su temporalidad propia y su duración es la misma" ". La retención mienta, por tanto, el seguir sabiendo ahora que recién ha sonado el sonido, aunque el material sonoro ya no pueda escucharse "en persona" y devenga cada vez menos claro a medida que el primer sonido va retrocediendo y siendo sucedido por otros y otros sonidos que emergen a cada nuevo instante. Es importante aquí distinguir en la retención lo retenido y el retener mismo. Cuando Husserl se refiere a la retención, pone el acento en el retener, es decir, no en el recuerdo del material percibido, sino en el hecho de que en el presente pervive retenido el haber percibido recién hace un instante el sonido. Es decir, en la conciencia de cada ahora pervive el haber sido consciente de los momentos inmediatamente pasados como tales, lo que posibilita que los objetos internos (los objetos tal cual aparecen a la conciencia) propiamente duren y que su fluir sea continuo. En efecto, si la conciencia se experimenta a sí misma ahora percibiendo un sonido incipiente, entonces los ahoras precedentes en los que la conciencia se experimentó a sí misma percibiendo otros sonidos que en el nuevo ahora ya no están dados, siguen presentes en el ahora actual como recién sidos, "y el trecho completo de la duración temporal desde el punto de inicio hasta el punto de ahora es consciente

${ }^{9} L F T$, p. 46 (Hua X, p. 24). 
como duración que ha transcurrido"10. En términos generales podemos decir que a lo largo de todo este río que se constituye en el pasar de un ahora a otro de la conciencia, el sonido es primero consciente en el modo de un ser esperado en tanto anunciado por los sonidos que le precedieron, es decir, en el modo de una protención; luego es consciente en el modo del darse en el ahora viviente, esto es, como impresión originaria, y después de haber sido escuchado todavía es consciente en la retención como habiendo recién sido. Este es el modo en el cual un objeto temporal inmanente -un tempo-objeto- aparece al fluir de la conciencia. El presente, por lo tanto, no es nunca un momento puntual, sino que es ex-tático, es un presente especioso que contiene junto con cada impresión originaria la protención de las fases futuras de la conciencia (y del tempo-objeto) y la retención de las pasadas. Impresión, retención y protención constituyen los protoactos de la conciencia del tiempo, es decir, aquellos a partir de los cuales la conciencia propiamente pasa de modo continuo de un ahora a otro, y posibilitan que en su flujo puedan durar los objetos. Hay que extender aquí a todos los protoactos la diferencia que introdujimos poco más arriba al referirnos a la retención, a saber, la diferencia entre el material hilético dado en los protoactos (lo retenido, protendido o percibido) y el modo de darse (el retener, protender o percibir mismo) ${ }^{11}$. Por un lado podemos dirigir la atención en la impresión, retención y protención sobre el objeto inmanente (lo percibido, retenido o protendido). Entonces captamos sus fases temporales y podemos afirmar, por ejemplo, que tal sonido está ahora durando con claridad y distinción, o que ha pasado y a medida que se aleja del sonido ahora incipiente lo recordamos con menor claridad, o incluso que los sonidos precedentes auguran y permiten anticipar un cierto sonido futuro que es cada vez menos claro cuanto más futuro. A esta dirección de la intención la llama Husserl intencionalidad transversal. Pero podemos también dirigir la mirada al modo en que somos conscientes de estas diferencias en el aparecer del objeto inmanente y su contenido de duración. Entonces captamos las fases temporales de la propia conciencia del objeto, y podemos afirmar que dentro de la distención de la duración del sonido, únicamente del punto de duración que está dado ahora podemos decir que

${ }^{10}$ LFT, p. 47 (Hua X, p. 24).

${ }^{11} \mathrm{Se}$ trata de una diferencia que habrá que tener constantemente presente porque en ella se cifra toda la problemática de la retención y del intento de fundar en ella el tiempo objetivo y su legalidad a priori. 
es percibido; de las fases pasadas del sonido decimos que son retenidas como recién o más lejanamente sidas, y de las fases futuras de su duración que son protendidas o esperadas. Se trata de la intencionalidad longitudinal. Precisamente el hecho de que en los protoactos la conciencia sea consciente en cada presente de su haber sido y de su estar por ser es lo que permite al objeto inmanente aparecer de un modo decursivo. Si sólo nos atuviésemos a las fases del objeto, entonces no habría propiamente hablando duración, sino superposición de fases diferentes que no podrían ser reconocidas como fases de un mismo objeto.

Ahora bien, ahondemos en la descripción husserliana de la constitución del continuo temporal, condición de posibilidad del aparecer decursivo de los objetos inmanentes. El decurso del tempo-objeto tiene un punto fuente. Se trata de aquella fase en la cual el objeto está dado perceptivamente a la conciencia. "El punto fuente que inaugura el 'producirse' del objeto que dura es una impresión originaria"12. Esta impresión originaria es lo que constituye en cada caso el "ahora". El ahora está en permanente modificación: el sonido que suena ahora cambia constantemente en la conciencia a retención de un sonido sido, y un sonido nuevo reemplaza al que ha pasado. El sonido retenido ya no se da perceptivamente, es decir, que carece de todo soporte hilético nuevo correlativo al acto retencional por el cual la conciencia capta su ser pasado, por lo que constituye una mera modificación de la impresión originaria: un recuerdo progresivamente menos claro (modificado) del contenido intuitivo de la impresión fontanal. Cada ahora está sometido a esta ley de la modificación. Envía al pasado al ahora anterior transformándolo en retención, hasta que el producirse de un nuevo ahora lo envía a él mismo al pasado como conciencia retencional nueva de lo que apenas recién fue conciencia impresional $y$, consecuentemente, la primera retención se transforma en retención de una retención. Y dado que el punto fuente se renueva constantemente, surgen nuevas retenciones y las retenciones previas se convierten en retenciones de retenciones precedentes. Por ello puede decir Husserl "que cada retención posterior no es mera modificación continuada que nace de la impresión originaria, sino modificación continuada de todas las modificaciones incesantes previas del mismo punto

${ }^{12}$ LFT, p. 51 (Hua X, p. 28). 
inaugural"13. De este modo, la serie de retenciones va formando un continuo en el que las retenciones más recientes van desplazando hacia el pasado las más antiguas y constituyendo el distanciamiento temporal ("la cola del cometa") respecto del punto fuente en constante renovación. Todo trecho o período de tiempo como así también toda fijación de un cierto punto fuente como presente no es sino un corte abstracto que el sujeto, por medio de un acto sintético y derivado de aprehensión, realiza en la primigenia continuidad incesante de este flujo retencional (y protencional). "Comenzar es comenzar a continuar"14. Esta descripción husserliana del continuo de modificaciones retencionales tiene una doble función. Por un lado dota al presente de densidad y extaticidad, por cuanto el continuo retencional como "cola de cometa" de cada nuevo punto fuente está también dado en el ahora actual, por lo que el presente se excede a sí mismo y desborda sobre el presente-pasado y el presente-futuro deviniendo "una continuidad en constante ampliación, una continuidad de pasados [retenidos y de futuros protendidos]"15. Por otro lado transfiere la intuitividad del presente al pasado reciente, aun cuando se trate de una intuitividad progresivamente degradada a medida que las retenciones se distancian del punto fuente y se convierten en modificaciones de modificaciones. La conjunción de estas dos funciones es la que permite reidentificar en cada ahora los múltiples y diferentes apareceres (escorzos) como escorzos de un único y mismo objeto inmanente ${ }^{16}$. El continuo retencional -el tiempo- es, para usar la expresión de Ricoeur, "un reto a la lógica de lo mismo y el otro"17. Durar es ser lo mismo transformándose en lo otro. Durar es persistir en el cambio.

\subsection{La noción de rememoración}

Las retenciones no alcanzan el conjunto del pasado. Su contenido intuitivo se va perdiendo hasta que se convierten en meras intenciones vacías. Para poder introducir en el continuo el pasado que efectivamente podemos recordar, pero que ya no puede ser retenido como cola de cometa

\footnotetext{
${ }^{13}$ LFT, p. 52 (Hua X, p. 30).

${ }^{14}$ Ricoeur, P., op. cit., p. 674.

${ }^{15}$ LFT, p. 51 (Hua X, p. 28).

${ }^{16}$ Cfr. LFT, p. 49 (Hua X, p. 26).

${ }^{17}$ Ricoeur, P., op. cit., p. 671.
} 
del presente impresional introduce Husserl la noción de rememoración, también denominada recuerdo secundario o reproductivo. La percepción se constituye sobre la base de sensaciones o datos hiléticos presentes en persona a la conciencia, la retención o recuerdo primario sobre la base de evocaciones o representaciones vicarias adheridas a las percepciones sidas. La rememoración o recuerdo secundario constituye, por el contrario, una representación que no está adherida al presente impresional por un continuo de modificaciones retencionales. Así, por ejemplo, con ocasión de escuchar una melodía nos acordamos de otra del mismo autor que hemos escuchado en otro concierto. No puede decirse que este recuerdo constituya el eco retencional de la melodía que acabamos de escuchar, puesto que se trata de otra melodía, sino de la re-producción o re-presentación de una percepción que tuvo lugar en un pasado que ya no se percibe como modificación retencional del actual punto fuente. Este recuerdo es precisamente la rememoración que se caracteriza, entonces, por volver a hacer presente $o$ presentificar en el ahora un objeto que ha sido percibido en el pasado y cuyo contenido intuitivo no está dado como modificación retencional del actual punto fuente. En tanto tal la rememoración implica la conciencia de haber sido percibido el objeto rememorado y se distingue de la mera fantasía (representación de objetos ficticios) porque en esta última "no se da ninguna posición del ahora reproducido, ni ninguna coincidencia de él con un ahora pasado"18. La rememoración, por el contrario, cree en el ser de lo rememorado, es decir, lo pone como percibido coincidentemente con un ahora pasado, y en virtud de esa posición lo localiza respecto del ahora actual. Aun cuando la rememoración se distingue de la mera fantasía, sigue siendo un acto intuitivo no perceptivo -es "como si" oyéramos la melodía, pero de hecho no la oímos- y no goza, por tanto, de la misma evidencia y claridad que la percepción. A pesar de ello lo intuido de modo no perceptivo está reproducido en el presente, es como si fuera presente, es un cuasipresente en el que se re-constituye ahora una objetividad duradera no dada en persona. Por ello mismo la intencionalidad propia de la rememoración guarda paralelismos notables con la de los protoactos, al punto que constituye una réplica (Gegenbild) suya. Así, análogamente a la impresión originaria, con la rememoración se presentifican también el haz de retenciones y protenciones que tuvieron como punto de origen ese recuerdo

${ }^{18}$ LFT, p. 72 (Hua X, p. 51). 
secundario. Sus retenciones, caídas en la indiferenciación, son vueltas a traer al presente "como si" hubieran sido recién percibidas, y sus protenciones, cuyo cumplimiento se dirige hacia el presente efectivo, son revividas como intenciones de expectativa que se han o no cumplido ${ }^{19}$. De modo igualmente análogo a la impresión originaria la rememoración pasa de impresión de una rememoración que está dándose a retención de una rememoración que acaba de haber sido y va perdiendo paulatinamente claridad. En síntesis, en la rememoración todo ocurre "como si percibiéramos de nuevo el objeto, pero sólo 'como si"' 20.Todo el proceso perceptivo es reproducido en la rememoración. sólo que lo recordado no es percibido de modo intuitivo ni como dándose ahora (impresión) ni como recién sido (retención), sino que es presentificado, vuelto a traer al presente, re-presentado. Esta falta de autodonación de lo recordado constituye la diferencia esencial entre recuerdo secundario por un lado y percepción y recuerdo primario por otro. La segunda diferencia que vale la pena aquí señalar es el carácter espontáneo de la rememoración, que es cuestión de la libertad, en oposición al carácter de afección de los protoactos, en cuanto la conciencia no puede salirse de su propio fluir. Una tercera diferencia radica en el grado de evidencia de la rememoración: lo retencionalmente consciente es absolutamente cierto (siempre en términos fenoménicos), mientras que es posible que lo representado o la secuencia a través de la cual se representa un suceso no haya sido percibida tal cual se la reproduce. La reproducción sólo puede conservar la misma evidencia que tiene la conciencia retencional generadora de tiempo por medio de un recubrimiento del curso reproductivo con el retencional, porque en este caso acaece una re-donación de lo reproducido. La noción de recubrimiento es decisiva porque compensa el corte entre la retención, que aún pertenece al presente, y la rememoración, que lo es del pasado, y refuerza la idea de continuidad temporal más allá de los límites del presente extático. Para terminar con nuestra reconstrucción de la noción de husserliana de recuerdo secundario digamos que tenemos que distinguir tres tipos. En primer lugar

${ }^{19}$ En realidad el cumplimiento en la conciencia rememorativa de una protención es un recumplimiento de una expectativa que ya se ha cumplido en el presente viviente; y si las protenciones originarias del recuerdo secundario eran indeterminadas, "en la rememoración tenemos una expectativa predirigida que no deja abierto nada de todo ello" LFT, p. 74 (Hua X, p. 53).

${ }^{20}$ LFT, p. 59 (Hua X, p. 37). 
aquella rememoración que hemos tomado como paradigma para la descripción del recuerdo reproductivo, a saber, el recuerdo retrospectivo (Rückerinnerung) que ilustra con un contenido intuitivo presentificado una retención vacía, es decir, una que ha caído en un completo punto de indiferenciación y perdido su vínculo con el presente viviente. En segundo lugar hay que mencionar la memoración de futuro $o$ espera (Zukunftserinnerung) que representa intuitivamente algo futuro como si ocurriese ahora. Así como el recuerdo retrospectivo ilustra una retención vacía, la memoración de futuro ilustra con una presentificación las protenciones vacías en cuanto no pueden ya predelinearse por las impresiones presentes. A su vez estas memoraciones de futuro sólo se cumplen, adquieren plena claridad y se plenifican por una donación en persona con la percepción ulterior. Finalmente hay que mencionar el recuerdo de presente (Gegenwartserinnerung) o memoración concomitante (Mitgegenwärtigung). Con la expresión recuerdo de presente Husserl se refiera a "la intuición por la que se presentifica algo sin tenerlo presente en persona como en el caso de la presentación o percepción" ${ }^{21}$; es decir, se trae al presente algo que no está dado como si estuviera dado en el presente. Los recuerdos de presente vendrían a ilustrar intenciones vacías que configuran un horizonte temporal ya no de sucesión, sino de simultaneidad. Una representación de esta índole sería, por ejemplo, la que mienta otro cuarto en el que yo no estoy ahora, pero que me represento como existiendo concomitantemente con la habitación que ocupo. Tal intención vacía se ilustra precisamente con la presentificación de una memoración concomitante 22 .

Esta breve y ciertamente incompleta ${ }^{23}$ reconstrucción de la naturaleza del recuerdo primario y el secundario no ha sido sino preparatoria para abordar la cuestión de la relación entre continuo retencional, por un lado, y el cuasi presente de la rememoración, por el otro. Esta es, en función de nuestra problemática, la cuestión central, porque para que las

21 Walton, Roberto, Husserl. Mundo, conciencia y temporalidad, Almagesto, Buenos Aires, 1993.

${ }^{22}$ Cfr. LFT, pp. 80-82 (Hua X, pp. 60-61).

23. Para un tratamiento detallado de la noción husserliana de recuerdo secundario, cf., Sonja Rinofner-Kreidl, Edmund Husserl: Zeitlichkeit und Intentionalität, Alber, Freiburg/München, 2000, pp. 275-287. 
rememoraciones puedan insertarse en un continuo temporal, en el cual Husserl funda la estructura a priori del tiempo objetivo, es menester reducir la retención a retención vacía, lo que, como veremos, genera graves problemas relativos al estatus fenomenológico del concepto de retención.

\subsection{Excedencia y circularidad}

La rememoración -dijimos- constituye una ilustración reproductiva de un continuo retencional vacío. En tal medida se inserta en la unidad de la corriente retencional vivida, y justamente por eso se puede hablar de historia y de una serie continua y prolongable de tiempo que va más allá de la caída del horizonte retencional del presente viviente en la indiferenciación. Pero para ello es necesario que la rememoración tenga valor ponente y no sea mera fantasía, es decir, que el pasado rememorado recubra un determinado ahora retencional pasado, que ha quedado vacío por su alejamiento del punto fuente actual. Tal recubrimiento se completa cuando el cumplimiento de las esperas de la rememoración conduce al presente viviente o retenido, es decir, cuando el presente realiza las anticipaciones de un pasado rememorado. Entonces es posible hablar de un "encadenamiento del tiempo en el que cada uno de los acontecimientos encuentra un lugar diferente" 24 . Ahora bien, para que tal recubrimiento efectivamente sea posible es preciso localizar la retención vacía recubierta por la rememoración, es decir, es preciso que la rememoración rememore también el ahora pasado (y vacío) del flujo de la conciencia del tiempo en el cual la conciencia remorativa pone como percibido su objeto y en virtud de esa posición lo sitúa respecto del presente efectivo. Husserl es absolutamente consciente de esta necesidad, por ello en un objeto temporal distingue, "por una parte, el contenido junto con su duración, que pueden ocupar uno u otro lugar en el orden 'del' tiempo, y, por otra parte, ésta su localización en el tiempo ${ }^{25}$ ". Por lo tanto en la rememoración debemos tener una intencionalidad doble: una dirigida al objeto en su duración, y otra concerniente a su localización. "Una duración no es representable en absoluto o, mejor, no es susceptible de posición si no es puesta en un orden del tiempo, si no existen intenciones referidas al orden del tiempo" 26 . Habría, pues, en la rememoración una

\footnotetext{
${ }^{24}$ Ricoeur, P., op. cit., p. 683.

${ }^{25}$ LFT, p. 74 (Hua X, p. 53).

${ }^{26}$ LFT, pp. 74-75 (Hua $X$, p. 53).
} 
duplicidad de intenciones según se dirijan a la reproducción del contenido intuitivo con que se dio el objeto duradero pasado o a su localización en el orden del tiempo. "Pero esta intención es no intuitiva, es 'vacía', y su objeto es la serie temporal objetiva de los acontecimientos, y esta serie es el entorno oscuro de lo actualmente rememorado". ${ }^{27}$ Esta segunda intencionalidad vacía de la rememoración es indispensable para constituir en la conciencia, por medio del recubrimiento de recuerdo primario y secundario. el encadenamiento objetivo del tiempo -la forma pura de la sucesión-, la cual ya no sería un presupuesto de nuestra conciencia, sino un correlato de intenciones dirigidas al lugar temporal y prescindentes del contenido rememorado. Tales intenciones son comprendidas por Husserl como trasfondo o halo oscuro que acompaña siempre a la intencionalidad reproductiva de un contenido intuitivo. La cuestión que, sin embargo, se plantea aquí es cómo es posible localizar y determinar objetivamente un ahora del flujo de la conciencia que, en tanto vacío, ha precisamente caído en la indiferenciación, es decir, que no es distinguible por la duración de ningún objeto de otro momento del flujo. En otros términos, cómo se puede afirmar fenomenológicamente que en el flujo constante e indiferenciado de la conciencia es posible la identificación de lugares fijos del flujo. En efecto, el lugar temporal identificado, si es que quiere afirmarse que el tiempo objetivo es producto de la conciencia y no presupuesto suyo, ha de ser un momento del flujo, es decir, un momento determinado generado por el continuo retencional. La segunda intencionalidad ha de estar dirigida, pues, a una retención. Y si es una rememoración vacía ha de estarlo a una retención vacía. Pero, precisamente, si la retención está vacía es indiferenciable de otra retención. Para escapar a la aporía de un continuo retencional que constantemente fluye pero que tiene lugares frjos, Husserl al final de la segunda sección se ve obligado a acudir a un concepto oscuro y problemático: el de "lugar de tiempo" (Zeitstelle). En la retención no sólo se retiene lo percibido en grados decrecientes de claridad, sino que se retiene el lugar de tiempo en que se percibió lo percibido y ese lugar de tiempo vacío, pero siempre igualmente claro, es el que la segunda intención de la rememoración puede reidentificar y lo que le permite constituir la forma pura de la sucesión.

${ }^{27} L F T$, p. 75 (Hua X, p. 54). 
En todo este complejo juego de intencionalidades dobles recubiertas se juega la cuestión central de las Lecciones, a saber, si luego de la desconexión del tiempo objetivo el análisis fenomenológico de la conciencia del tiempo interno puede mostrarse como fundamento constitutivo del tiempo desconectado. En este juego la noción de "lugar de tiempo" es una carta decisiva. Según Husserl, frente al flujo siempre en retroceso de modificaciones retencionales no sólo el objeto que aparece distanciándose hacia el pasado es conservado aperceptivamente en identidad absoluta (es decir, las apariciones en retroceso son percibidas como continuidad de escorzamiento de un objeto), sino que, a la par, se conserva "la posición que experimentó en el punto de ahora como "éste"" 28 . "A la esencia del río modificante pertenece el que cada lugar de tiempo se ofrezca idéntico y como necesariamente idéntico"29. Cada ahora actual, junto con una impresión fenoménica, dona un lugar de tiempo, "que es algo muy esencialmente distinto frente a la cualidad y frente a los restantes momentos materiales del contenido de sensación" ${ }^{30}$. El ahora donado conserva paradójicamente su lugar en el retroceso continuo del fenómeno hacia el pasado, es decir, se conserva paradójicamente fijo como el mismo ahora en el fluir temporal, sólo que adquiere carácter de pasado en cuanto la impresión que le dio origen retrocede respecto del surgimiento de un nuevo ahora. La identidad del objeto en su devenir temporal (del tempo-objeto) no descansa, entonces, básicamente sobre el contenido material de la sensación (que la primera intencionalidad de la rememoración puede reproducir invariado), sino también de los lugares fijos de tiempo ocupados por esta sensación a lo largo del flujo (a los que se dirige la segunda intencionalidad de la rememoración). En efecto, el mero contenido de la sensación perteneciente a los diferentes puntos actuales de un objeto puede ser reproducido de modo tan invariado como se quiera, pero no individualiza el objeto en su duración si no se tiene en cuenta que la misma sensación ahora y en otro ahora entraña una diferencia que corresponde al lugar absoluto de tiempo en el que ella se dio. Es la retención de este lugar, diferente del contenido hilético de la impresión, la fuente primigenia que permite identificar el tempo-objeto así aparecido en cierto momento o, como dice Husserl, como "éste". Por ello puede afirmar el filósofo que es el lugar de

\footnotetext{
${ }^{28}$ LFT, p. 85 (Hua X, p. 65).

${ }^{29}$ LFT, p. 86 (Hua X, p. 66).

${ }^{30}$ LFT, p. 88 (Hua X, p. 68).
} 
tiempo "el que viene primeramente a constituir individualidad" 31 , en cuanto permite recubrir constantemente un contenido con un punto fijado en el flujo. Esta capacidad de retener los puntos o lugares fijos de tiempo cuando fluye hacia el pasado la impresión que los deparó es condición necesaria, pero aún no suficiente para que la temporalidad del flujo adquiera las características de unidad, homogeneidad y continuidad que constituyen el tiempo objetivo. Para que ello ocurra juega un papel determinante el recuerdo reproductivo que gracias a su segunda intencionalidad puede individualizar y presentificar una y otra vez el lugar o punto de tiempo retenido de modo vacío y gracias a su primera intencionalidad presentificar su contenido intuitivo. Cuando ello ocurre se abre el propio halo de retenciones de ese lugar de tiempo reproducido y una nueva rememoración puede reidentificar el lugar de tiempo de las retenciones del primer momento reproducido y presentificar su respectivo contenido intuitivo, abriéndose así un nuevo campo retencional, ahora del segundo momento reproducido, y así continuamente. De este modo voy reconstruyendo la ordenación unitaria, serial y homogénea del tiempo por el continuo recubrimietno de las retenciones longitudinales vacías de los lugares temporales abiertos por la presentificación operada por un primer recuerdo secundario con la presentificación reproductiva de dichas retenciones por una segunda rememoración ${ }^{32}$. Este proceso puede prolongarse indefinidamente permitiendo el paso del presente extático a tiempo histórico que supera la memoria de cada uno.

Que el propio Husserl no queda conforme con su respuesta a la pregunta acerca del origen del tiempo objetivo en el juego complejo de superposiciones de intenciones retencionales y rememorativas dobles se transparenta ya en el hecho de que, apenas él ha respondido a esta cuestión, se vuelve a preguntar " ¿cómo se funda en ello [en el solapamiento de los campos de tiempo] la ordenación lineal según la cual cualquier segmento de tiempo, también los reproducidos fuera de toda continuidad con el campo de tiempo actual, ha de ser parte de una única cadena que prosigue ininterrumpidamente hasta el ahora actual?" 33 . Y es que la respuesta de Husserl deja muchas cuestiones abiertas. En primer lugar, no queda claro

\footnotetext{
${ }^{31}$ LFT, p. 87 (Hua X, p. 67).

${ }^{32}$ Cfr. LFT, p. 90-91 (Hua X, p. 70-71).

${ }^{33}$ LFT, p. 91 (Hua X, p. 71).
} 
que es propiamente un "lugar de tiempo" y cómo es posible que la conciencia retencional pueda retener un lugar separadamente de su contenido -cuestión absolutamente necesaria si el lugar es identificado como tal luego de la caída en la indiferenciación del contenido. Husserl reconoce que el lugar de tiempo se origina con el manar de cada nueva impresión, por lo tanto debería indiferenciarse con ella, sin embargo no lo hace. Cómo es posible que continúe fijo, cuando aquello en función de lo cual él es el lugar que es fluye hacia la indiferenciación, es algo que Husserl no explica. En segundo lugar, y en estricta correlación con lo anterior, no se entiende cómo puede la conciencia rememorativa distinguir por su forma vacía un lugar de otro cuando se ha indiferenciado su contenido. Dicho de otro modo, lo incomprensible es cómo puede la rememoración adjudicar el lugar de tiempo correcto (reproducir la intención vacía de la retención) al contenido que presentifica si no hay nada (o nada claro) que permita individualizar los lugares de tiempo. Husserl tiene razón al decir que la individualización del lugar de tiempo es condición de la individualización del tempo-objeto, pero no explica cómo ni sienta las condiciones que hagan posible en el propio flujo de la conciencia individualizar el lugar de tiempo. En tercer lugar, la introducción de lugar de tiempo complica la diferencia entre retención y rememoración. No se ve en qué difiere la segunda intencionalidad de la rememoración, que rememora una intención vacía, de la retención que retiene vacío el lugar de tiempo. Ambas apuntan a una misma intención vacía, y no puede decirse que la rememoración presentifique algo, porque justamente la intención es vacía. Por otra parte, la noción de lugar de tiempo, indispensable, por un lado, para encadenar el tiempo, vuelve, por otro, insuficiente la distinción entre rememoración ponente y retención. En efecto, si los lugares temporales son retenidos, cualquier rememoración se haya siempre conectada al presente por la retención vacía de la cadena de lugares temporales, cuando lo propio de la rememoración era su desconexión respecto del ahora actual. Este problema es patente cuando se rememora un recuerdo aún fresco. ¿Cómo distinguir allí si se trata de la presentificación de un contenido intuitivo ya no dado o de la continuidad de la presencia de lo dado en persona? Y es menester que esta diferencia esté clara, porque gracias a la superposición de los campos retencionales y las reproducciones puede constituirse el encadenamiento objetivo. Si ante el recuerdo del paso del tren no puedo determinar si se trata del recuerdo fresco del tren que acaba de pasar o de la presentificación de un presente ya no dado, no puedo saber si el campo de retenciones que 
ese recuerdo abre sigue siendo el del presente actual o el de uno que ya no es actual y que, por tanto, se extiende más hacia el pasado.

Todas estas dificultades menores revelan los dos problemas de fondo que padece el intento husserliano de fundar el tiempo objetivo en el flujo de la conciencia: la excedencia y la circularidad. El análisis husserliano de las Lecciones no ha tenido en cuenta la excedencia del tiempo objetivo respecto de lo reproducible en base a rememoraciones y esperas. En efecto, a menos que se niegue que el universo y su transformación precede a todo acto de una conciencia individual, habrá que aceptar que el tiempo le pasaba a los entes con anterioridad a cualquier continuo retencional. De ese paso quedan testimonios bajo formas de las huellas materiales del paso del tiempo indagadas por las ciencias de la naturaleza, por ejemplo, los fósiles, los estratos geológicos o los procesos entrópicos analizados por la termodinámica. Y a menos que se niegue que la muerte, como dijimos, instaura un después de cada conciencia individual y no un después en la conciencia, habrá que aceptar que el tiempo, más allá de toda conciencia, sigue afectando el curso del universo. Hay un después que le sigue pasando a los objetos pero que no puede ser jamás rememorado por la conciencia individual. Pero esta excedencia no es sólo cuantitativa, sino cualitativa. El curso homogéneo del tiempo objetivo es independiente de y no se doblega a la constitución de la temporalidad de la conciencia. De ello no hay mejor testimonio que la senescencia corporal que, para usar la expresión de Levinas, "va cociendo a fuego lento" 34 todo intento rebelde de la conciencia humana de adueñarse del todo, adueñándose del tiempo. La decrepitud del cuerpo, como todas las otras huellas materiales del paso del tiempo, constituyen los reinscriptores a través de los cuales la excedencia cuantitativa y cualitativa del tiempo objetivo se ofrece a ser aprehendida por la conciencia humana, sin por ello hallar en ella su origen ni su forma. Sin embargo, metodológicamente puede negarse esta excedencia, puede negarse el mundo trascendente, pero entonces surge el segundo problema central del análisis husserliano, a saber, el que sea evidente a la conciencia el fenómeno de la retención vacía de lugares temporales. Husserl necesita suponer estos lugares para constituir el flujo de la conciencia como un encadenamiento objetivo, pero en ningún momento describe en las Lecciones -y un análisis

${ }^{34}$ Levinas, Emmanuel, De otro modo que ser o más allá de la esencia, trad. A. PintorRamos, Sígueme, Salamanca, 1987, p. 106 
fenomenológico debería haberlo hecho- cómo se muestran estos lugares. Suponer su necesidad conceptual no implica en absoluto su autodonación fenomenológica. Que sea un dato que cuando recordamos no sólo recordamos algo, sino que una segunda intencionalidad del recuerdo recuerda un lugar temporal fijo y vacío, que, en tanto tal, está conceptualmente abstraído del flujo del contenido impresional que le dio origen, es altamente cuestionable. El lenguaje aquí más que en ningún otro punto de las Lecciones deja de ser descriptivo para construir a priori un concepto abstracto -el de "lugar temporal"vinculado a la "forma de la sucesión- que le permita adjudicar al fluir de la conciencia las características que se presuponen propias del tiempo objetivo. Ricoeur nuevamente tiene razón cuando afirma que "todo sucede como si la idea de sucesión lineal única sirviera de guía teleológica para buscar y encontrar, en la relación entre la intencionalidad segunda de la representación y la intencionalidad primera [longitudinal] de la retención, una aproximación lo más estricta posible a la idea de sucesión lineal" 35 . Esa presuposición de las características del tiempo objetivo en el subjetivo es notoria cuando Husserl en el \# 33 de las Lecciones le atribuye a la constitución del flujo como evidentes y a priori todas las leyes -unicidad, unidad, continuidad, homogeneidad, transitividad- propias del tiempo objetivo. Y lo hace fundándose "en la validez de las evidencias fundamentales sobre el tiempo que son captables inmediatamente, y que resultan evidentes sobre la base de las intuiciones en que se dan los lugares de tiempo"36. Es decir, funda la evidencia de la atribución de estas leyes a priori a la constitución del flujo por el juego de intencionalidades retentivas y rememorativas de una intuición vacía, la cual en absoluto es evidente. El problema de fundar una evidencia en algo que a todas luces no resulta evidente: en una intuición de algo indescifrable y sin contenido intuitivo como es un lugar de tiempo (ya la expresión "lugar de tiempo"es una expresión metafórica resultante de espacializar el tiempo vivido y no la descripción de una intuición) hace pender sobre las Lecciones la amenaza de la circularidad. La constitución del tiempo subjetivo que fundamenta las leyes a priori del tiempo objetivo presupone en realidad la legalidad que fundamenta. $\mathrm{El}$ análisis del tiempo desemboca así en un verdadero círculo del tiempo. La amenaza no es menor, porque si el tiempo objetivo está presupuesto, la desconexión anunciada en el punto de partida queda

35 Ricoeur, P., op. cit., p. 689, nota 30.

${ }^{36}$ LFT, p. 91, (Hua X, p. 71). 
impugnada, y la excedencia, fácticamente experimentable en los que hemos llamado reinscriptores, pero metodológicamente puesta entre paréntesis, regresa para dar cuenta de lo limitado del análisis fenomenológico. El tiempo parece no querer encerrarse en una conciencia, ni aún cuando fuese absoluta.

\section{La conciencia absoluta del tiempo}

En la tercera sección de las Lecciones, Husserl advierte que es necesario que la conciencia del tiempo sea absoluta, es decir, que se autoconstituya y se automanifieste, si ha de hallarse en ella la fuente fenomenológica originaria y constitutiva de todas las formas del tiempo, porque si ella fuese constituida o evidenciada por un acto de reflexión se podría siempre preguntar por el transcurso de la conciencia en la que ese acto tiene lugar, y la regresión al infinito se volvería inevitable. Hay, por lo tanto que distinguir tres planos. Primero, el del tiempo objetivo desconectado, la duración de los objetos trascendentes. Segundo, el de la duración de las vivencias u objetos inmanentes, de la se ocupa la segunda sección y que acaece como una continuidad de escorzos a través de la cual un mismo objeto se manifiesta en proceso de cambio o de reposo o en la alternancia entre lo uno y lo otro. Tercero, el flujo absoluto de la conciencia, su constante discurrir, en el cual es posible toda duración. Las unidades del segundo plano pertenecen todavía a los fenómenos constituidos, puesto que la duración no es intrínseca del objeto, sino del fenómeno en cuanto correlato de una conciencia que fluye y cuyo fluir hace del aparecer del objeto una continuidad de escorzamiento. El tercer plano, por tanto, pertenecerá al orden de los fenómenos constituyentes del tiempo. Tales fenómenos son por naturaleza distintos de las unidades objetivas constituidas en el tiempo. Es posible decir de estas últimas que son simultáneas o sucesivas, que se suceden en ellas fases de cambio y de reposo, que existen ahora o que no han existido antes, porque se dan como continuidad de escorzos para el curso de una conciencia que está constantemente fluyendo y generando en su fluir, desde cada impresión originaria, un continuo de modificaciones retencionales y protencionales. No puede decirse de este flujo constituyente que cambie, puesto que no puede dejar de fluir, ni que sea simultáneo con otro flujo, pues todo fluye en él, ni que exista ahora y no haya existido antes, sino la serie de apariciones de los tempo-objetos sería discontinua. En consecuencia, no será posible apoyarse en los predicados atribuídos a la 
descripción de la duración de los tempo-objetos para describir el flujo de la conciencia constituyente de esa duración. No podemos decir para describir la continuidad del flujo que se da ahora y que se ha dado en un ahora anterior o se dará en uno posterior. A lo sumo podemos decir que el ahora actual pertenece al flujo constituyente del tiempo que lo hace surgir como ahora por la impresión originaria y que el ahora pasado pertenece (y no que perteneció) a ese mismo fluir que lo constituye retencionalmente como dado en el pasado. Pero si es así, ¿no es entonces el flujo también una secuencia con un ahora y una serie de ahoras previos dados retencionalmente? A esta pregunta responde Husserl que éste es un modo metafórico de describir el flujo en función de lo constituido por él y no en función de sí mismo, puesto que él no es una secuencia en el tiempo, sino que genera el tiempo. Sin embargo, es un modo inevitable. El propio Husserl lo admite: "Este flujo, este río es algo que denominamos así según lo constituido, pero que no es nada objetivo en el tiempo. Es la subjetividad absoluta y tiene las propiedades absolutas de lo que en imagen designamos como 'flujo', 'río', como algo que brota ahora en un punto (...) que es fuente primigenia, etc. (...). Para todo esto nos faltan los nombres"37. En este momento del análisis cabría preguntarse si la necesidad de recurrir a imágenes (flujo, punto fuente, etc. ) o de describir lo constituyente por lo constituido (fase presente, continuidad de pasados, modificación, etc.) no es un indicio claro de la imposibilidad de mostrar y, consecuentemente, describir el tiempo en sí mismo. Y, además, describiendo lo constituyente por lo constituido, se corre el riesgo de reducir el análisis de la conciencia absoluta al mero translado de vocabulario, por obra del cual los análisis considerados primero en términos de aparición de objetos trascendentes reducidos a vivencias, serían retomados luego en términos de conciencia ${ }^{38}$. En el fondo reaparece aquí el problema antes señalado: el de la presuposición de la forma del tiempo objetivo y la consecuente determinación por él de la subjetividad absoluta. De otro modo ¿cómo sabríamos que a pesar de la multiplicidad de impresiones originarias $y$ de sus series de respectivos continuos retencionales que empiezan y se hunden en la indiferenciación, todas ellas responden "a una forma que las vincula en la medida en que la ley de transformación del ahora en ya-no y, por otra parte, del aún-no en ahora, aplicándose a todas las series, (...), rige (...) una igualdad general en el modo

${ }^{37}$ LFT, p. 95 (Hua X, p. 75).

${ }^{38}$ Cfr. Ricoeur, P., op. cit, p. 690. 
de fluir"39; cómo sabríamos que múltiples sensaciones originarias existen simultáneamente, y que "cuando una de ellas fluye, fluye 'a la vez'la muchedumbre de las restantes, y lo hace en modo enteramente igual, con gradaciones enteramente iguales (...)" 40 ; cómo sabríamos, en fin, que a pesar de la multiplicidad de conciencia, y de los múltiples modos en que los sujetos viven la distención temporal de un mismo suceso, "el tiempo inmanente se constituye como uno único para todos los objetos y sucesos inmamentes" 41 ? Y la crítica puede ir aún más allá. Si el tiempo -el problema de la falta de nombres pareciera en esto dar razón a Kant- no es accesible en sí mismo, sino por la transformación del mundo entero que constituye su testimonio, y si los testimonios del paso del tiempo exceden, como vimos antes, los lapsos vivenciables y constituibles por una conciencia, entonces hasta qué punto es sostenible que la conciencia es el origen absoluto del tiempo.

Sin embargo Husserl reafirma la prioridad constituyente del tiempo inmanente respecto del objetivo constituido, y declara que en el flujo único de conciencia se constiuyen a la vez la unidad temporal del objeto inmanente y la unidad del propio flujo de conciencia ${ }^{42}$. Si no se quiere caer en la mentada regresión al infinito, suponiendo otra conciencia que constituya la unidad del flujo con las mismas características que la unidad del flujo constituye la unidad temporal de los objetos inmanentes, es necesario admitir, "por chocante que parezca" 43 , que la conciencia, constituyendo la forma y unidad de la duración de los objetos inmanentes, constituye a la vez la propia forma y unidad de su flujo. Y que concomitantemente con la experiencia de la duración de las vivencias experimentamos marginalmente la duración de la conciencia que constituye aquella otra duración. En efecto, como el propio Husserl advierte, puede parecer chocante, "si es que no absurdo en principio" 44 , sostener que lo constituyente es al mismo tiempo y en el mismo acto lo constituido, pues "la conciencia interna no tiene como correlato a datos inmanentes que duren, sino que tiene a las fases

\footnotetext{
${ }^{39}$ LFT, p. 96 (Hua X, p. 77).

${ }^{40}$ Ibid.

${ }^{41}$ LFT, p. 97 (Hua X, p. 77).

${ }^{42}$ Cfr. LFT, p. 100 (Hua X, p. 80).

${ }^{43}$ Ibid.

${ }^{44}$ Ibid.
} 
constituyentes de esas unidades" 45 , es decir, es en sí misma y al mismo tiempo constituyente y constituido. Pero el filósofo se limita a declarar "así es como ocurre en efecto" 46 . En este estrato último de la conciencia absoluta del tiempo, contra el esquema fundamental de la fenomenología que distingue en el acto intencional el correlato noético constituyente y noemático constituido, los dos correlatos son aquí lo mismo: constituyendo la unidad temporal de las vivencias, la conciencia constituye su propia unidad. La originalidad del "tercer plano", el del flujo absoluto, consiste, pues, en sumar a la desconexión de la duración de los objetos trascendentes la desconexión de la duración de los objetos inmanentes (vivencias) constituida por el propio flujo de la conciencia, para, así, formalizar las relaciones entre los protoactos que forman el flujo considerados en sí mismos. Pero allí surge la pregunta de cómo es posible vivenciar los protoactos en sí mismos, cómo es posible que se manifiesten los protoactos sin el sostén de alguna objetividad constituida. Dicho de otro modo: ¿cómo es posible que haya una impresión o una protención o una retención que no lo sea de algo? ¿Cómo es posible esta segunda desconexión? Husserl se ve obligado a apelar a un desdoblamiento de la intencionalidad de los protoactos. Una primera intencionalidad se dirige hacia el tempo-objeto retenido y otra hacia la conciencia que es consciente de sí misma como habiendo sido recién consciente. Sin embargo, por más que tenga todos los visos de serlo y que sea incluso difícil describirla sin generar la sospecha de que lo sea, esta segunda intencionalidad no es un acto de reflexión sobre la retención, sino que se da o vivencia conjuntamente con ella. "El tránsito de una percepción del objeto a una modificación retencional [del objeto] y el tránsito del percibir a una modificación retencional del percibir se copertenecen con necesidad" 47 . Por el primer tránsito (intencionalidad transversal) se constituyen las fases del objeto inmanente que dura. Por el segundo (longitudinal) la ordenación de las fases del flujo. La primera es una temporalidad fenoménica o inmanente; la segunda una cuasi-temporalidad (porque todo dura en el flujo, pero el flujo no dura, sino que es siempre el mismo) prefenoménica o protoinmanente que "se constituye intencionalmente como forma de la conciencia tempoconstituyente y lo hace

\footnotetext{
${ }^{45}$ LFT, p. 141 (Hua X, p. 118).

${ }^{46}$ LFT, p. 100 (Hua X, p. 80).

${ }^{47}$ LFT, p. 141 (Hua X, p. 118).
} 
en la propia conciencia" 48 . La unidad temporal inmanente del objeto y la unidad del flujo de la propia conciencia serían, pues, procesos análogos y concomitantes, por lo que se podrían retomar todos los análisis de los protoactos en términos de flujo y no de tempo-objeto. Pero con ello se genera una nueva paradoja: ¿cómo es posible que la conciencia, que constituye la duración fenoménica, se dé a sí misma en el mismo acto constituyente la misma forma de la duración sin por eso durar (porque la conciencia absoluta es cuasi-temporal)? Además de la paradoja citada, siguen sin responderse ninguno de los cuestionamientos que habíamos formulado más arriba relativos a la evidencia de esta intencionalidad longitudinal, introducida ya en la distinción hecha en la segunda sección entre lugar temporal y las fases del decurso del objeto. Sigue, en efecto, sin quedar claro que fácticamente tengamos, por más que sea de modo marginal, una experiencia de la duración del flujo en sí mismo, a partir de la cual determinemos la forma de la duración de los objetos inmanentes, y de los objetos sin más, en cuanto la duración de los objetos trascendentes se edifica sobre la continuidad de escorzamiento de las vivencias inmanentes. En términos ricoeurianos: “¿puede bastarse a sí misma la conciencia de evidencia de la duración sin la de una conciencia perceptiva?"49 ¿Hasta qué punto puedo afirmar que el flujo en sí mismo autoparece de un cierto modo si es inescindible de la aparición de la duración de los objetos inmanentes, pues, como el propio Husserl declara, las dos intencionalidades se dan inseparablemente unidas 50 ? $\mathrm{Y}$ "necesariamente tiene que producirse un autoaparecer del flujo"51 en sí mismo y no a través de lo fluyente, si es que éste quiere ser fenomenológico y absoluto, y no el resultado de la transposición constructiva al flujo de la forma de darse del tiempo objetivo externo $^{52}$. La sospecha de tal transposición se incrementa, porque hay que

\footnotetext{
${ }^{48}$ LFT, p. 103 (Hua X, p. 83).

${ }^{49}$ Ricoeur, P., op. cit., p. 692.

${ }^{50}$ Ibid..

51 Ibid.

${ }^{52}$ Conviene aclarar aquí una vez más el sentido de nuestra crítica, para evitar malas interpretaciones. No negamos, en principio, la tesis husserliana según la cual la conciencia no es solamente consciente del objeto, sino que se autoexperimenta siendo consciente protencional, impresional o retencionalmente. $Y$ ello no por un acto de reflexión o reproducción posterior, sino concomitantemente con la conciencia del objeto. En este sentido hay que darle la razón a Husserl en que si la
} 
reconocer a esa segunda intencionalidad retencional, un carácter absolutamente pasivo ${ }^{53}$ para que la espontaneidad del yo no altere la forma única y una del flujo. Por este camino llega Husserl a afirmar años después una dimensión "del absoluto y primigenio pre-ser del fluir" 54 , supuesta por toda actividad espontánea del yo, a las que habría que desconectar para acceder a esta dimensión originaria del fluir en su pasividad originaria. Finalmente termina elaborando la noción de un "fluir sin yo (icbloses Strömen)" como un estrato último y primigenio que es a la vez "intemporal" y "portador de todas las temporalizaciones y tiempos" 55 . ¿Pero el paradojal tener que recurrir primero a una intencionalidad, es decir, a un acto, absolutamente pasivo, y finalmente a un "fluir sin yo", es decir, a una temporalidad subjetiva sin sujeto, en la que la conciencia constituyente no puede en rigor constituir nada más que lo que ya está dado de un cierto modo con independencia del yo, no es el testimonio más claro de que no es la conciencia quien constituye la forma del flujo, sino que ella se somete a un tiempo que la trasciende? El cuerpo, que padece la senescencia, dolorosamente refrenda esta sospecha.

conciencia de las fases del objeto no fuese a la vez protoconciencia, es decir, si no fuese originariamente consciente de ser conciencia impresional y sólo lo fuese por rememoración o reflexión, "sería incomprensible qué es lo que presta a esta fase [la impresión] la cualificación de 'ser ahora."' LFT, p. 142 (Hua X, p. 119). "Y es esta protoconciencia la que transita a la modificación retencional -que es entonces retención de ella y del dato originariamente consciente en ella, pues ambas retenciones son inseparablemente una-: si no existiese protoconciencia, tampoco sería pensable retención ninguna; la retención de un contenido inconsciente es imposible." (ibid.). Todo ello es admisible. Lo que aquí se pone en cuestión es que sea evidente que esa experiencia del flujo (que es inescindible de la duración del objeto y que no puede darse por sí misma) manifieste por sí misma y en sí misma una cierta forma, y que esa forma constituya el tiempo objetivo. Antes bien, la forma en que duran los objetos trascendentes reducidos a vivencias es aquello a partir de lo cual se comprende la forma del flujo. El flujo no se muestra por si mismo ni somos conscientes a priori de cómo fluye en sí mismo el flujo. A lo sumo puede decirse que somos conscientes de que el flujo fluye y el cómo de su fluir está determinado por el modo en que fluye en él lo que fluye, y no a la inversa.

${ }^{53}$ Cfr. LFT, p. 108 (HuaX, p. 88)

${ }^{54}$ Hua XV : Zur Phänomenologie der Intersubjektivität. Texte aus dem Nacblaß. Dritter Teil : 1929-1935, Hrsg. Von Iso Kern, 1973, p. 585.

${ }^{55}$ Hua XV, pp. 598, 584, 587. 


\section{Aporética}

El problema, al que repetidas veces hicimos mención, de tener que apoyarse para describir la legalidad a priori del tiempo subjetivo constituyente en la experiencia a posteriori de la legalidad del tiempo objetivo constituido, no mienta meramente una circularidad lógica o una debilidad soslayable del análisis, sino que señala el carácter aporético de las dos formas del tiempo que no pueden reducirse la una a la otra. En efecto, no es posible reducir el tiempo objetivo a la temporalidad subjetiva. Y ello por la mencionada excedencia cuantitativa y cualitativa del primero, es decir, porque su ámbito de concernimiento va más allá de todo lo constituible por la conciencia: la muerte, la senescencia, en una palabra, la transformación del cosmos, van acaeciendo más allá de todo proceso constitutivo de la conciencia. El tener que vivenciar (en el propio cuerpo o a través de huellas) estas efectividades testimonia la sobrepotencia del tiempo, cuyo correlato es la finitud: la imposibilidad de todo absoluto, $y$, ante todo, de la conciencia absoluta del tiempo. $\mathrm{Y}$ es que no es la conciencia (individual o intersubjetiva) quien origina el tiempo, sino el tiempo quien termina con la conciencia. No es tampoco la conciencia quien determina el modo en que pasa el tiempo, sino que el modo en que pasa el tiempo va de-terminando las posibilidades de la conciencia, hasta terminar con todas ella. Y, finalmente, no son las series de tiempo quienes se hunden en la indiferenciación de la conciencia, sino la conciencia quien se hunde en la indiferenciación del tiempo. De este hundimiento, de esta angustiosa finitud, de este saber que el mar infinito de infinitas horas nos tragará, ha tenido siempre conciencia el hombre. Y la entera historia de la literatura, que una y otra vez retoma el topos de la angustia por el sentido indescifrable del paso del tiempo, da cuentas de ello.

Pero si el tiempo objetivo no se deja reducir a la temporalidad de la conciencia, tampoco la temporalidad subjetiva, el modo en que la conciencia discurre, se deja reducir al tiempo objetivo. En efecto, este último no puede dar cuenta de los fenómenos de distensión y concentración propios de la temporalidad subjetiva pura. Cada uno de ellos es respectivamente puesto a la luz por aquellos dos temples señalados y radicales que denominamos angustia y esperanza. Angustia y esperanza son temples señalados y radicales porque constituyen autorreferencias afectivas de la conciencia a su propio discurrir. Tales autorreferencias, en tanto mientan la temporalidad subjetiva 
pura, no se refieren a la temporalización de un objeto inmanente, es decir, no aluden a la vivencia de la duración de un objeto, la cual se concreta como la proyección protencional de un horizonte de futuro y la retroyección de un horizonte de pasado en el presente especioso de la impresión originaria, en el que se sincronizan los horizontes temporales. En otras palabras, los temples radicales no ponen en evidencia la temporalidad extático-horizontalsincrónica de la conciencia, correlativa a la duración de un objeto inmanente, sino que son los dos modos en los que la conciencia experimenta su propio discurrir puro. Así, la angustia, que como bien lo ha observado Heidegger, no es angustia por un ente, sino por nada, por nuestro propio ser en el mundo que no es ente alguno, sino en esencia temporalidad, acaece cuando se disuelven en la nada, en la absoluta insignificación e indiferenciación, todas las percepciones. Entonces el existente experimenta, por un momento, que es, que discurre. Dicho de otro modo, experimenta no el paso del tiempo (el tiempo en sí mismo es inasible), sino su puro pasar en el tiempo. Pero después de que el minutero del reloj haya dado tal vez un cuarto de vuelta, del mar de indiferenciación emerge y cobra relieve de nuevo un ente. Una percepción saca al existente de la inmersión angustiosa en su propio decurso. Objetivamente medido, el momento de angustia duró quince minutos. Pero esos minutos se vivieron (fueron una vivencia no reflexiva) como una duración sempiterna. Se trata de una duración sin horizontes, porque en la angustia nada hay desde lo cual retro- o pro-yectar un horizonte. De una duración no sincrónica, porque en ella no hay pasado retenido ni futuro protendido en el presente, menos aún rememoraciones, sino el continuo e inacabable durar de un mismo momento indiferenciado, vacuo y homogéneo. De una duración no extática, porque el presente no desborda hacia sus horizontes, porque no hay nada que dure a no ser la duración misma. Se trata, finalmente, de una duración distendida, porque ella distiende en un momento el conjunto de la duración de la conciencia. En efecto, en la angustia profunda el existente no se proyecta a otro instante ni reconoce horizonte ninguno, todo su tiempo se haya para él absorbido por ese instante que, siendo el mismo, dura continuamente, y que no reconoce otro después que la muerte. El instante que en la angustia sigue al instante de angustia es la muerte, pues nada experimenta el angustiado entre el uno y la otra. La angustia produce así una distensión del tiempo: en un lapso objetivo se vivencia la totalidad de la duración de la conciencia. Ésta específica forma de vivenciar la propia temporalidad es incomprensible en la dimensión del tiempo objetivo, en el 
cual los instantes no se distienden, no duran sempiternamente, sino que responden a las leyes que Husserl quiso adjudicarle al discurrir de la propia conciencia. La angustia es, pues, el testimonio de que no sólo la vivencia del propio discurrir de la conciencia (la automostración del fluir) no responde, contra lo que pensaba Husserl, a la legalidad apriorística de la sucesión lineal y homogénea de lugares temporales, sobre la base de la cual se constituiría el tiempo objetivo, sino que la propia dimensión objetiva del tiempo no puede dar cuenta de esta específica forma del discurrir inmanente. $\mathrm{Y}$ a la angustia se opone esa otra autorreferencia afectiva radical de la conciencia a un modo en que se da su propio discurrir, que es la esperanza. La genuina esperanza no es la espera de un objeto, sino la afección de un instante eterno. En la esperanza el existente vivencia anticipadamente en el ahora un estar ligado a un instante futuro (escatón) en el cual se concentra sin durar la totalidad absolutamente plena de su decurso temporal. Lo peculiar de esta ligazón es que no hay un fluido por el cual me halle protencionalmente ligado con ese instante, sino que lo que establece el vínculo es un salto, por el cual la conciencia se siente ya en relación con un instante más futuro que todo advenir desde el presente, con un instante futuro respecto del tiempo en el que duran los objetos inmanentes. Se trata, en el caso de la esperanza, de un in-stante que incurre puntualmente en el curso siempre sincronizable de las vivencias y que se renueva una y otra vez, pero sin durar - no estamos en un instante de esperanza más cerca de lo esperado que en el anterior-. Precisamente por ello no se deja sincronizar. En efecto, el in-stante de esperanza representa un quiebre con todo continuo retencional y no remite a ningún futuro que podamos protender, justamente porque en él no hay ningún objeto que pudiese escorzarse en horizontes. En la esperanza religiosa la conciencia no espera ningún objeto sólo experimenta, incurriendo en su propio discurrir, la plenitud de todo su tiempo concentrado en un instante no durativo: aquel en el que todos los tiempos del sujeto pasan a ser uno. El modo en que la conciencia siente su temporalidad cuando es conciencia esperanzada representa una reversión respecto del modo en que discurren en ella los objetos inmanentes cuando es conciencia perceptiva. Es el futuro supratemporal quien irrumpe en el presente de la conciencia, no para dejar un continuo retencional, sino para concentrar en el instante la totalidad del tiempo: hacer resplandecer la eternidad. Esperanza y angustia, autorreferencias afectivas radicales de la conciencia a su temporalidad pura, muestran que el tiempo objetivo no puede dar cuenta del subjetivo y que la vivencia de la temporalidad de la 
conciencia no sólo no origina el tiempo objetivo, sino que no puede describirse sobre la base del decurso de este tiempo ni responde a sus leyes a priori.

Pero si el tiempo de la conciencia y el tiempo objetivo no se dejan reducir uno al otro, tampoco pueden darse uno con independencia del otro. El tiempo de la conciencia es abarcado por el tiempo objetivo que le pasa, y el tiempo objetivo no puede ser asido sin reconvertirlo ipso facto en tiempo de la conciencia. Dicho de otro modo: hay conciencia del tiempo porque hay un tiempo que pasa, pero, a su vez, el tiempo sólo efectivamente pasa en cuanto se es consciente de su pasar. Esta aporía, este reenvío constante de un tiempo al otro, está a la base de los análisis husserlianos y de sus dificultades. Ella representa el círculo que amenaza con encerrar todo quijotesco intento por atrapar el tiempo.

\section{Resumen}

Este artículo analiza las Lecciones de fenomenología de la conciencia interna del tiempo. En primer lugar se propone mostrar la excedencia del tiempo cósmico respecto de la temporalidad de la conciencia. En segundo determinar si la descripción husserliana de la forma del flujo de la conciencia absoluta del tiempo es circular, es decir, si no presupone el tiempo objetivo que pretende constituir. En tercero someter a análisis crítico la automostración del flujo absoluto de la conciencia. Finalmente se menciona la aporética que se cierne sobre toda investigación que quiera reducir uno forma del tiempo a la otra o asirlas independientemente.

Palabras clave: "Tiempo", "objetividad", "subjetividad", "circularidad".

\section{Abstract}

The article examines Husserl's work On the Phenomenology of the Consciousness of Internal Time. Firsttly, it seeks to show the surplus of cosmic time with regard to the temporality of consciousness. Secondly, an attempt is made to establish whether the Husserlian description of the form of the flow of consciousness is circular, i.e., whether it does not presupposes the objective time that it purports to constitute. Thirdly, a critical analysis of the self-manifestation of the absolute flow of consciousness is advanced. Finally, a reference is made to the aporetics that befalls any investigation that tries to reduce one form of time to the other or to apprehend them independently.

Key words. "Time", "objectivity", "subjectivity", "circularity". 